This is a final peer-reviewed accepted manuscript.

The final form has been published in Food chemistry Volume 294, 1 October 2019, Pages 260-266

The final published version is available online at: https://doi.org/10.1016/j.foodchem.2019.05.047

2

\title{
Investigation of water state during induced crystallization of honey
}

Silvia Tappi ${ }^{1}$, Luca Laghi ${ }^{12^{*}}$, Amanda Dettori ${ }^{2}$, Lucia Piana ${ }^{3}$, Luigi Ragni ${ }^{1,2}$, Pietro Rocculi ${ }^{1,2}$

${ }^{1}$ Interdepartmental Centre for Agri-Food Industrial Research, Alma Mater Studiorum, University of Bologna, Piazza Goidanich 60, 47521 Cesena (FC), Italy

${ }^{2}$ Department of Agricultural and Food Science, Alma Mater Studiorum, University of Bologna, Campus of Food Science, Piazza Goidanich 60, Cesena (FC), Italy

${ }^{3}$ Piana Ricerca e Consulenza, Castel San Pietro Terme, Bologna.

* Corresponding author. E-mail address: to 1.laghi@unibo.it

\section{Abstract}

This work studied water state of honey during crystallization, obtained statically and dynamically, by differential scanning calorimetry (DSC), water activity $\left(a_{\mathrm{w}}\right)$ assessment and time domain nuclear magnetic resonance (TD-NMR).

Crystallization was induced by adding $5 \%$ of crystallized honey to three honey samples with different fructose/glucose ratio, the key characteristic for honey crystallization. Samples were stored at $14{ }^{\circ} \mathrm{C}$. Dynamic crystallization was obtained by using an impeller. DSC showed that the dynamic crystallization was faster than the static one, the latter characterized by two phases, showing different rates. The crystallization rate did not affect $\mathrm{a}_{\mathrm{w}}$, that remained below 0.600. TD-NMR allowed to separately observe two kinds of protons, both pertaining to liquid sugars, one chemically exchanging with water and one not exchanging with it. The combination of techniques allowed speculating that the two crystallization methods led to crystals of different size and shape.

\section{Keywords}

Differential scanning calorimetry; dynamic crystallization; Honey; static crystallization; time-domain nuclear magnetic resonance; water activity; water state

(C) 2019 Elsevier. This manuscript version is made available under the Creative Commons AttributionNonCommercial-NoDerivs (CC BY-NC-ND) 4.0 International License (http://creativecommons.org/licenses/by-nc-nd/4.0/) 


\section{Introduction}

27 Honey is a supersaturated solution that contains mainly glucose and fructose (70-80\%) and only small amounts of other sugars. The crystallization, or granulation, of honey is a natural phenomenon that occurs during storage and involves only glucose, as fructose is characterized by a higher solubility value.

The rate of crystallization depends on many factors, among which amount of glucose, fructose and water, temperature, glucose supersaturation level, viscosity and presence of pre-formed crystals or impurities (Conforti, Lupano, Malacalza, Arias, \& Castells, 2006; Venir, Spaziani, \& Maltini, 2010). Nucleation can be classified as primary or secondary. Primary nucleation occurs when the system does not contain any pre-formed crystal and an energy barrier has to be overcome for the formation of new nuclei. Collision among molecules in the solution leads to the formation of clusters that, if sufficiently big, can overcome the energy barrier and become stable (Hartel, 1993). Higher supersaturation levels increase the probability for clusters to overcome the critical dimension. The secondary nucleation can occur only when pre-existing crystals are present. A secondary crystal can be generated from dendritic growth on the surface of a primary nucleus or when a primary nucleus collides with another primary nucleus or with other components of the system, such as the walls of the vessel (Hartel, 1993).

Guided or induced static crystallization $(1,987,893,1935)$ is based on the secondary nucleation phenomena and involves the introduction of fine seed crystals that will act as primary crystallization nuclei into liquid honey. Such procedure on one side allows to obtain finely granulate honey, on the other side avoids both unpredictable changes in the texture of honey during storage and crystallization defects (Dettori, Tappi, Piana, Dalla Rosa, \& Rocculi, 2018).

Dynamic crystallization (Gonnet, 1994) consists in carrying out the guided crystallization under a slow manual or automatic stirring of the mass for a few days, to impart creaminess and spreadability to the crystallized product. Honey obtained in this way is defined as creamy honey and its peculiar rheological characteristics are due to the formation of very small crystals (Karasu, Toker, Yilmaz, Karaman, \& Dertli, 2015). The crystallization rate and rheological characteristics of honeys have been investigated by many works in relation to composition and crystallization levels (Slavomir Bakier, 2007; Sławomir Bakier, Miastkowski, \& Bakoniuk, 2016; Conforti et al., 2006; Dobre, Georgescu, Alexe, Escuredo, \& Seijo, 2012; Venir et al., 2010). However, to the best of our knowledge, the differences between static and dynamic crystallization have never been investigated before. 
In addition, the works published on honey have rarely addressed the behavior of water during crystallization, beyond the mere observation of water activity (Gleiter, Horn, \& Isengard, 2006; Zamora \& Chirife, 2006). Water activity determines honey microbiological stability during storage. It is deeply influenced by crystallization because glucose binds six molecules of water in liquid honey, but crystallizes mainly in the monohydrate form. Crystallization thus promotes water concentration in the liquid phase, leading in turn to an increase of water activity, allowing the growth of osmophilic yeasts (Gleiter, Horn, \& Isengard, 2006).

DSC measurements have been successfully applied to evaluate the crystallization of honey by various authors (Al-Habsi, Davis, \& Niranjan, 2013; Venir et al., 2010), who measured the amount of glucose crystals on the basis of their melting enthalpy. Transverse relaxation time $\left(\mathrm{T}_{2}\right)$ of the protons observed by TD-NMR has been found able to give precious information about water interaction with solutes and sample's structures in several food matrices (Mauro et al., 2016; Petracci et al., 2012). These interactions have been also studied in honey during crystallization, where they have even been used to assess product adulterations through water state ( Ribeiro et al., 2014; Ribeiro et al., 2014).

The aim of the present study is to apply differential scanning calorimetry (DSC), water activity and time domain nuclear magnetic resonance (TD-NMR) measurements to investigate the behavior of water in honey during induced crystallization carried out in a traditional static manner or during a dynamic process, achieved through constant stirring of the mass at the optimal crystallization temperature $\left(14^{\circ} \mathrm{C}\right)$.

\section{Material and methods}

76

\section{Raw material and preparation of static crystallization samples}

The honey samples used in the present study were selected with the aim of having specific F/G ratios of approximately 1.05, 1.20 and 1.40, leading to fast (FC), medium (MC) and slow (SC) crystallization, respectively (Dettori et al., 2018). Before the experiment of crystallization kinetics, samples were gently heated up to $50{ }^{\circ} \mathrm{C}$ to melt any pre-formed crystal. The absence of glucose crystals was evaluated by optical microscopy. The crystal nuclei used were obtained by citrus honey finely granulated, added to the three samples so to reach $5 \%$ of the total mass. The so obtained mix was manually stirred with a spatula at room temperature for $10 \mathrm{~min}$. Samples were analyzed for water (at 20 ${ }^{\circ} \mathrm{C}$ with an Abbe refractometer) and sugars content (DIN-NORM-10758, 1997). During static crystallization, sampling itself could break the crystalline structure. To limit this confounding factor, 
liquid honey added with crystal nuclei and stirred was poured into samples holders ready for each analytical determination. Samples, created in triplicate, were stored in a climatic chamber at $14{ }^{\circ} \mathrm{C}$ throughout the entire crystallization process. Storage time needed for complete crystallization of each sample was assessed by means of preliminary tests. Sampling intervals were then adjusted accordingly, as follows: 0, 2, 7, 9, 22, 43 and 50 days for the FCs samples; 0, 10, 15, 34, 51 and 62 days for the MCs samples; 0, 7, 14, 21, 28, 34, 41, 48, 63, 83 and 102 days for the SCs samples.

\section{Raw material and preparation of dynamic crystallization samples}

Samples were subjected to dynamic crystallization with an in-house made steel temperature controlled stirrer, equipped with a helical impeller having a diameter of $100 \mathrm{~mm}$ and rotating at $14 \mathrm{rpm}$. The mixing chamber (about 1.2 L of volume) was externally cooled with a flux of water/ethylene glycol fluid, so to grant a stirred sample temperature of $14{ }^{\circ} \mathrm{C}$. To create the samples, liquid honey at room temperature was added with 5\% (w/w) of crystallized honey and placed in the stirring chamber. Every sample took 3 to 4 hours to reach $14{ }^{\circ} \mathrm{C}$. The moment when the $14{ }^{\circ} \mathrm{C}$ were reached was considered as $\mathrm{T}_{0}$ for the analysis. Samples were collected from the stirring chamber without interrupting the process. Storage durations were determined for each sample through preliminary tests and were of 10, 16 and 32 days for samples FCd, MCd and SCd, respectively. Sampling intervals were 0, 1, 2, 4, 7, 8 and 10 days for the FCd samples; 0, 1, 2, 3, 5, 7, 9, 12, 14 and 16 days for the MCd samples; 0, 2, 3, 4, 7, 9, 11, 14, $18,21,25,28$ and 32 days for the SCs samples.

\section{Differential Scanning Calorimetry}

Thermal analysis was carried out by differential scanning calorimetry using a DSC Q20 (TA Instruments, Germany) equipped with a cooling unit (TA-Refrigetated Cooling System90). Heat flow and temperature calibration were performed with distilled water $\left(\mathrm{T}_{\mathrm{m}} 0.0{ }^{\circ} \mathrm{C}\right)$ and indium $\left(\mathrm{T}_{\mathrm{m}} 156.60\right.$ ${ }^{\circ} \mathrm{C}$ ) under a dry nitrogen flow of $50 \mathrm{~mL} \mathrm{~min}^{-1}$.

Honey samples were weighed in $50 \mu \mathrm{l}$ aluminium DSC capsules and sealed. At each sampling time, three replicates were analyzed through temperature scanning at $5{ }^{\circ} \mathrm{C} / \mathrm{min}$ from 14 to $100{ }^{\circ} \mathrm{C}$.

Peaks were integrated with the Software TA-Universal analyzer, determining melting temperature $\left(T_{m}\right)$ and enthalpy $(\Delta \mathrm{H})$ of the granulated honeys.

\section{Water activity $\left(\mathbf{a}_{\mathrm{w}}\right)$}

Water activity was measured with an ACQUA LAB Water Activity Meter, (Decagon Devices, US). 
116 For statically crystallized honey, three samples holders were filled with liquid honey at the beginning

117 of the storage, then measured at each sampling time. Between measurements, samples were covered 118 with lids and protected with parafilm. For dynamically crystallized honey, samples were collected at 119 each sampling time.

\section{TD-NMR}

121 The transverse relaxation time $\left(\mathrm{T}_{2}\right)$ of protons was measured at $25^{\circ} \mathrm{C}$ with the Carr-Purcell-Meiboom122 Gill (CPMG) (Meiboom \& Gill, 1958) pulse sequence ( Ribeiro et al., 2014; Ribeiro et al., 2014), using 123 a Bruker Minispec PC/20 spectrometer (Bruker, Germany) working at $20 \mathrm{~Hz}$. The exponential decay 124 comprised 3200 echoes, an echo time (TE) of $0.080 \mathrm{~ms}$, leading to a dead time of $167 \mu \mathrm{s}$, and a recycle 125 delay of $5 \mathrm{~s}$. The number of scans and the amplification factor were chosen so that a $\mathrm{S} / \mathrm{N}$ ratio value of 126300 was reached, while signal clipping was prevented. Data mining was performed in R computational 127 language (R Development Core Team, 2011), by means of routines developed in-house. In order to 128 treat honey as a two components system, by following de Ribeiro et al. (Ribeiro, Mársico, Carneiro, Monteiro, Júnior, et al., 2014), the phased experimental curves were fit towards the sum of two exponential decays, according to the equation:

$$
S_{(t)}=\sum_{i=1}^{N} I_{n} e^{\left(\frac{-t}{T_{2, n}}\right)}+E_{(t)}
$$

131 where $I_{n}$ represents the intensity of each proton population and $T_{2, n}$ its transverse relaxation time.

132 The presence of two water populations postulated by Ribeiro et al. could be doubted by the massive 133 work by Brown (Brown, 1989), who demonstrated that the sum of two exponential curves fit nicely 134 also the signal of a single water population. This happens when water covers a wide range of relaxation 135 rates, a common case in food matrices (Iaccheri et al., 2015; Laghi et al., 2005; Petracci et al., 2014), 136 thus giving the false impression of two water components. For this reason the $\mathrm{T}_{2}$ decays, centered and 137 scaled to unity variance, were also employed to build a robust principal component analysis (rPCA) 138 (Hubert, Rousseeuw, \& Vanden Branden, 2005) model. This was done by setting an alpha value of 139 0.75. For this model, we calculated the scoreplot, the projection of the samples in the PC space, tailored 140 to highlight the underlying structure of the data. Besides, we calculated the Pearson correlation plot, 141 relating the concentration of each variable to the model components. 


\section{Statistics}

143 Differences among samples at specific time-points were looked for by anova test, with Tukey as a post144 hoc test, by taking advantage of the aov function of the R package "stats" (Chambers, Freeny, \& 145 Heiberger, 1992).

146 For TD-NMR data, differences in the overall trends characterizing the FCs, MCs and SCs samples 147 along storage time were looked for by two-way anova test, followed by Tukey as a post-hoc test. The 148 limited number of samples per point was considered by applying the tests on ranks (Conover \& Iman, 149 1981). Intensity and $T_{2}$ of each proton population and score values in the rPCA (Hubert et al., 2005) 150 model registered along time were interpolated by means of non-parametric functions. For the purpose, 151 a local regression model was applied, by taking advantage of the loess function (Cleveland, Grosse, \& 152 Shyu, 1992) of the R package "stats", with degree of smoothing equal to 0.9 and the degree of the 153 polynomial equal to 1.

\section{Results and discussion}

\section{DSC}

156 The precise composition (after crystal nuclei addition) of the samples fast (FCs), medium (MCs) and 157 slow (SCs) static crystallization is reported in Table 1. Slight differences were observed between each 158 of the couples of samples grouped as FC, MC or SC. However, the selection allowed to obtain very 159 similar fructose/glucose ratio, the parameter the mostly affecting the crystallization rate.

160 The detailed evolution of the melting enthalpy during static and dynamic crystallization is reported in

161 figure 1, while overall features of the crystallization processes are reported in table 2.

162 The melting enthalpy is proportional to the amount of crystallized glucose, so that at $\mathrm{T}_{0}$ its value 163 reflects the amount of finely crystallized honey added as starter. FC, MC and SC samples stored 164 statically reached the maximum crystallization values of $34.72,27.62$ and $21.68 \mathrm{~J} / \mathrm{g}$, respectively. 165 These values were proportional to the glucose supersaturation level, hence to the amount of glucose 166 that could crystallize (Dettori et al., 2018). Glucose supersaturation level determined also the 167 crystallization rate, so that FCs, MCs and SCs samples reached the maximum melting enthalpy in 50, 16890 and 102 days, respectively.

169 Although dynamic crystallization is known to increase granulation rate, to our knowledge, no previous 170 study has actually compared the crystallization behavior of honey according to static and dynamic 171 crystallization process. Results obtained showed for the first time that the dynamic process boosted 
172 significantly the crystallization rate, which was 5 to 6 fold faster than the static counterpart. In detail,

173 full crystallization was reached in 10, 16 and 35 days for FCd, MCd and SCd samples, respectively.

174 This was expected, as the nucleation process (both primary and secondary) is known to be strongly

175 increased by inputs of energy into the system, here represented by the mechanical energy given by

176 continuous mixing. According to Hartel (Hartel, 1993), the reason is that an external energy input 177 promotes random energy fluctuations, represented by local concentrations of sugar exceeding the 178 critical value for nucleation. Moreover, agitation promotes the forced migration of molecules, so to 179 reduce the hindrance to mass transfer given by viscosity.

180 It is possible to notice from figure 1 that, in statically stored samples, the crystallization kinetic showed 181 a linear trend along the entire process, but with an inflection occurring after 9, 15 and 21 days in FCs, 182 MCs and SCs samples, respectively, corresponding to the $50-60 \%$ of the process. A similar result was 183 observed by Venir et al. (Venir et al., 2010) in taraxacum honey. In particular, they observed a change 184 of slope after the crystallization of $15 \%$ of glucose, corresponding to the $60 \%$ of the total glucose that 185 could undergo crystallization.

186 Following Serra-Bovehì (Serra-Bonvehì, 1974), the two stages observed by us and Venir et al. (2010) 187 could be ascribed to the alternation of nucleation and crystal growth. The two phenomena can occur 188 simultaneously, but at different rates in relation to the supersaturation level. At the beginning of the 189 process, when supersaturation is high, the formation of new crystals is faster than their growth. As the crystallization proceeds, the nucleation rate decreases exponentially, so that, in a second stage, the 191 predominant process is the enlargement of the existing nuclei.

192 The two-phase behavior could not be observed in honey samples crystallized dynamically, that instead 193 showed a linear increase of melting enthalpy along the entire storage time. This observation could be 194 rationalized by considering that the energy input represented by the stirring promotes the formation of 195 nuclei, while the constant movement of the mass inhibits the excessive growth of the crystals.

196 Hence, the different crystallization method adopted not only noticeably influenced the crystallization 197 rate, but it also promoted changes in the formation of crystals that is at the basis of the difference in the 198 rheological properties, as described by Gonnet (1994).

199 Water activity

200 Figure $\mathrm{S} 1$ reports the evolution of $\mathrm{a}_{\mathrm{w}}$ in honey samples statically and dynamically stored as a function 201 of melting enthalpy. Initial values ranged between 0.490 and 0.550 . Differences were likely to be 202 caused by the different concentration of sugars and water in the honey samples. Indeed, as shown in 
203 table 1, water content varied in the $16.0-17.7 \%$ range. Values of $\mathrm{a}_{\mathrm{w}}$ increased systematically with the 204 amount of crystallized glucose. In detail, the observed changes in $a_{w}$ were in the 0.3-0.6 range, in 205 agreement with previous investigations (Zamora \& Chirife, 2006) carried out on 49 different honey 206 samples. However, in all cases, the final value never exceeded 0.60, the threshold usually considered 207 for osmophilic yeast growth.

208 Contrarily to the melting enthalpy, the increase in $\mathrm{a}_{\mathrm{w}}$ followed a linear trend for statically stored 209 samples, with no evident change of slope. This could be explained by considering that water activity 210 depends on the overall characteristics of the samples, failing to discriminate fine differences in the state 211 of water throughout the sample itself.

\section{TD-NMR: two components model}

213 By following the works of Ribeiro et al. (Ribeiro et al., 2014; Ribeiro et al., 2014), T 2 weighted TD214 NMR signals were fit to a model postulating the possibility to separately observe two protons pools, 215 that were named $T_{21}$ and $T_{22}$ (Fig. 2). Analysis of variance showed that, for both $T_{2}$ and intensity of the 216 two populations, level of supersaturation and time had in each case a statistically significant effect $217(\mathrm{p}<0.001)$. To grab the trends of the two populations along storage in a non-parametric fashion, 218 smoothing trends were calculated. The main features of the so evidenced trends are summarized in 219 table 3.

220 In agreement with Ribeiro et al. works, the two protons pools had, at $\mathrm{T}_{0}$ in the statically crystalized 221 samples, $\mathrm{T}_{2}$ values around 1.5 and $5 \mathrm{~ms}$ respectively. Their relative intensities were found in the 222 present work to be around 55\% and 45\%. Ribeiro et al. ascribed the two populations exclusively to 223 water differently interacting with crystals, but such ascription seems very unlikely, because it does not 224 consider the remarkable number of protons of the sugars. A few qualitative considerations drive the 225 point.

226 In liquid honey, each mole of water brings 0.11 moles of protons. Each mole of glucose or fructose is 227 characterized by 0.028 moles of protons pertaining to -OH groups. Around $75 \%$ of them (0.021 moles) 228 was found to be labile (Fabri, Williams, \& Halstead, 2005). The exchange rate of these protons 229 between water and sugars is expected (B. Hills, 1998; Venturi et al., 2007) to be much higher than the 230 NMR signal registration rate, reasonably far above $100 \mathrm{~s}^{-1}$ (Fabri et al., 2005). In this regime, water and 231 labile sugar protons are observed as a single population. Such population can be simply called 232 "exchangeable", as suggested by Petracci et al. (Petracci et al., 2014) on a different matrix. The 
remaining sugar protons bound to carbons plus the non-labile $-\mathrm{OH}$ protons (accounting for 0.046 moles) cannot exchange with water, so that they can be called "non-exchangeable".

According to the above considerations, in the samples we have analyzed in the present work, nonexchangeable and exchangeable protons populations may contribute to $\mathrm{T}_{2}$ weighted TD-NMR signals with about a 50:50 relative intensity. Such qualitative consideration is in very good agreement with the ratio measured in SC samples (51.47:48.53), while it shows a 17.14\% discrepancy in the case of FC samples.

The pool of exchangeable protons is expected to have longer $T_{2}$ values, according to the following reasoning. The $\mathrm{T}_{2}$ of a proton pertaining to liquid sugar is reasonably in the range of milliseconds. When this proton is exchanged between sugar and water its $T_{2}$ is the weighted average of the $T_{2}$ of the two sites, according to the Carver and Richards (Carver \& Richards, 1972), corrected by Hills and coworkers (B. P. Hills, Wright, \& Belton, 1989). Such $\mathrm{T}_{2}$ is undoubtedly longer than the one of nonexchangeable protons, because water has been found liquid even in a glassy matrix (B. P. Hills \& Pardoe, 1995), what translates into $T_{2}$ in the range of hundreds of milliseconds. According to this consideration, we therefore suggest that the populations originally named $\mathrm{T}_{21}$ and $\mathrm{T}_{22}$ by Ribeiro et al. can be ascribed to non-exchanging and exchanging protons, respectively.

Along the entire storage period the two protons pools model was able to fit nicely the $\mathrm{T}_{2}$ weighted signals registered on every sample, even on those where crystallization has occurred massively. This suggests that the liquid fraction of honey had the major, if not the exclusive, contribution to the NMR signal. Indeed, the CPMG pulse sequence we employed had an unavoidable dead time of $167 \mu$ s. The protons pertaining to the crystals were expected to be largely unobservable, because characterized by a $\mathrm{T}_{2}$ of a few microseconds (B. P. Hills \& Pardoe, 1995). Interestingly, as already observed for the increase of melting enthalpy, for each of the studied samples two distinct stages of crystallization could be noticed from a $T_{2}$ point of view. However, the change was observed at different times. The first stage could be considered as complete at days 30, 50 and 66 for FCs, MCs and SCs samples, respectively. During this stage, the relative intensity of nonexchangeable protons population decreased by $8.7 \%, 7.6 \%$ and $4.6 \%$ in the FCs, MCs and SCs samples, respectively. In parallel, the $\mathrm{T}_{2}$ of non-exchangeable protons increased by $74 \%$ to $200 \%$, while the $\mathrm{T}_{2}$ of exchangeable protons increased by $10 \%$ to $45 \%$. The main contribution to the trends of both relative populations and $\mathrm{T}_{2}$ values in the first stage is very likely the subtraction of glucose from the liquid fraction due to crystallization, leading to an increased concentration of water, as noticed by 
265 the exchangeable protons in the system $(\approx 53 \%)$. As confirmation, the FCs samples, with a higher 266 supersaturation index, showed also the largest and quickest increase of exchangeable protons, followed, 267 proportionally, by MCs and SCs samples. Moreover, the increase of water concentration in the liquid 268 fraction has two further effects. First, it moves the weighted average of $T_{2}$ of the exchangeable protons 269 towards higher values. Second, it increases the tumbling rate of the molecules, leading to higher $T_{2}$ 270 values for both exchangeable and non-exchangeable protons (Bordoni et al., 2014).

271 The second stage of static crystallization highlighted by $T_{2}$ weighted signals started around day 30,50 272 and 66 for samples FCs, MCs and SCs, respectively. This stage was characterized by a partial inversion 273 of the previous trends, with an increase of non-exchanging protons and with a shortening of the $T_{2}$ 274 values of both populations. The most likely rationalization of this phenomenon is that the crystals were 275 so densely spread across the entire honey volume that a high percentage of the still liquid molecules 276 interacted with them. Even if it is not possible to describe rigorously such interaction, it probably 277 comprised a reduced mobility, leading to shorter $T_{2}$ values, and a less effective sugar-water protons 278 exchange, that increased the number of non-exchanging protons. A confirmation seems to be devised in 279 the fact that the highest reduction of $\mathrm{T}_{2}$ values and exchanging protons occurred in the FC samples, 280 characterized by the highest amount of crystallized sugar, what translates into a higher solid-liquid 281 interface. In addition, at the liquid-solid interface local gradients of the magnetic fields form, thus 282 leading to shorter $T_{2}$ values (Dunn, 2002), linked to the dimension and the shape of the crystals.

283 Dynamically crystalized samples did not show appreciable differences from the statically crystalized 284 counterparts at $T_{0}$. The dynamic crystallization process showed the same overall features of the first 285 stage of static crystallization, with an increased concentration of exchangeable protons and an increased $286 T_{2}$ values for both populations for each level of glucose supersaturation. The remarkable feature of 287 these trends is the entity of the changes. While the first stage of static crystallization, when crystal 288 nucleation is favoure, leads to a decrease in the pool of non-exchangeable protons of $8.7 \%, 7.6 \%$ and $2894.6 \%$ for FC, MC and SC samples respectively, dynamic crystallization leads in the same samples to a 290 reduction of $9.8 \%, 15 \%$ and $5.8 \%$. Differences even more clear could be noticed for $\mathrm{T}_{2}$ values. As an 291 example, while the $\mathrm{T}_{2}$ of non-exchangeable protons increased for FC, MC and SC samples by $80.9 \%$, 29256.9 and $26.4 \%$ as a consequence of static crystallization, the same values increased by $198.2 \%$, $293114.3 \%$ and $74 \%$, respectively, as a consequence of dynamic crystallization. Interestingly, stirring 294 made the values of dynamically crystallized MC and SC samples change similarly to FC samples. This 295 suggests that at the base of the phenomenon is the number of crystals, which it is higher in the samples 296 crystallized dynamically. 


\section{TD-NMR: model-free analysis}

298 In order to employ $\mathrm{T}_{2}$ weighed NMR signals to gain information on the samples without applying a 299 priori determined model, robust principal component analysis (rPCA) (Hubert et al., 2005) was applied 300 on the centered and scaled signals points (Figure 3). In the scoreplot (Figure 3A), the samples spread 301 with storage time along PC 1, which represented $96.8 \%$ of the total samples variance.

302 The samples that the two components model identified as collected at the end of the first stage of static 303 crystallization appeared at negative values along PCA, while samples freshly prepared or collected at 304 the end of crystallization were characterized by high and intermediate scores, respectively. This made 305 the pattern covered by the samples along PC 1 undoubtedly similar to those highlighted by the two 306 protons pools model. Again similarly to the two protons pools model, the samples collected at the end

307 of the dynamic crystallization were located at scores that were far lower than the corresponding created 308 with static crystallization. The correlation between the points of the $T_{2}$ weighted signals and their 309 importance over PC1 (Figure 3C) showed, in agreement with the two components model, that in the 310 fresh samples the non-exchangeable protons played the highest role, while the opposite was observable 311 at the end of the first stage of crystallization.

312 The non-parametric approach constituted by the rPCA model directly calculated on the signals 313 registered by TD-NMR confirmed, from a protons $\mathrm{T}_{2}$ point of view, that the static crystallization could

314 be divided into two stages, the second of which partly reversing the effects of the first one. From a $\mathrm{T}_{2}$ 315 point of view, the interactions between crystals and liquid honey seemed of similar type but of different 316 extent for statically and dynamically crystallized samples.

\section{Conclusions}

318 The water behavior in honey during induced crystallization according to static and a dynamic process 319 was investigated.

320 DSC measurements confirmed that the constant movement of the honey during storage decreased the 321 time necessary for the complete crystallization of all the honey types by 5-6 fold. Static crystallization 322 showed two main phases of crystal genesis, identified both by DSC and TD-NMR measurements, 323 characterized by different rates, probably related to the nucleation and crystal growth phases 324 alternation. On the contrary, dynamic crystallization was characterized by a linear trend that was 325 attributed to a prevalence of the nucleation phenomenon over the growth of crystals. Moreover, the 326 crystallization rate did not influence the $\mathrm{a}_{\mathrm{w}}$ increase, that remained below 0.600 . 
Through TD-NMR two populations of protons were identified and attributed to liquid sugars protons

328 exchanging and non-exchanging with water. The interaction between crystals and liquid honey showed some differences according to the type of crystallization process adopted, that could be due to the different number and size of the crystals. However, further investigation is necessary to confirm this 331 hypothesis.

332 In general, the described multi-analytical approach confirmed the suitability of the different techniques to study the water mobility in differently crystalized honey, giving integrative results able to increase 334 the knowledge of these complex phenomena with a different level of detail.

\section{Acknowledgements}

336 The authors declare no conflicts of interest

\section{Bibliography}

Al-Habsi, N. A., Davis, F. J., \& Niranjan, K. (2013). Development of Novel Methods to Determine Crystalline Glucose Content of Honey Based on DSC, HPLC, and Viscosity Measurements, and Their Use to Examine the Setting Propensity of Honey. Journal of Food Science, 78(6). https://doi.org/10.1111/1750-3841.12103

Bakier, S. (2007). Influence of temperature and water content on the rheological properties of polish honeys. Polish Journal of Food and Nutrition Sciences, 57(2 (A)), 17-23. https://doi.org/10.1590/S151635982010000800027

Bakier, S., Miastkowski, K., \& Bakoniuk, J. R. (2016). Rheological properties of some honeys in liquefied and crystallised states. Journal of Apicultural Science, 60(2), 153-166. https://doi.org/10.1515/JAS-2016-0026

Brown, R. J. S. (1989). Information available and unavailable from multiexponential relaxation data. Journal of Magnetic Resonance (1969), 82(3), 539-561. https://doi.org/10.1016/0022-2364(89)90217-5

Carver, J. P., \& Richards, R. E. (1972). A general two-site solution for the chemical exchange produced dependence of T2 upon the carr-Purcell pulse separation. Journal of Magnetic Resonance (1969), 6(1), 89- 
Chambers, J. M., Freeny, A., \& Heiberger, R. M. (1992). Analysis of variance; designed experiments. In Statistical Models in S (pp. 145-193). Wadsworth and Brooks/Cole Advanced Books and Software, Pacific Grove, California.

Cleveland, W. S., Grosse, E., \& Shyu, W. M. (1992). Local regression models. Statistical Models in S, 2, 309 376. https://doi.org/10.2307/1269676

Conforti, P. A., Lupano, C. E., Malacalza, N. H., Arias, V., \& Castells, C. B. (2006). Crystallization of honey at $-20^{\circ} \mathrm{C}$. International Journal of Food Properties, 9(1), 99-107. https://doi.org/10.1080/10942910500473962

Conover, W. J., \& Iman, R. L. (1981). Rank transformations as a bridge between parametric and nonparametric statistics. American Statistician, 35(3), 124-128. https://doi.org/10.1080/00031305.1981.10479327

Dettori, A., Tappi, S., Piana, L., Dalla Rosa, M., \& Rocculi, P. (2018). Kinetic of induced honey crystallization and related evolution of structural and physical properties. $L W T, 95,333-338$. https://doi.org/10.1016/j.lwt.2018.04.092

DIN-NORM-10758. (1997). Analysis of honey; determination of the content of saccharides fructose, glucose, saccharose, turanose and maltose; HPLC method. Deutsches Institut für Normierung (1997--05) Berlin; Germany.

Dobre, I., Georgescu, L. A., Alexe, P., Escuredo, O., \& Seijo, M. C. (2012). Rheological behavior of different honey types from Romania. Food Research International, 49(1), 126-132. https://doi.org/10.1016/j.foodres.2012.08.009

Dunn, K. J. (2002). Enhanced transverse relaxation in porous media due to internal field gradients. Journal of Magnetic Resonance, 156(2), 171-180. https://doi.org/10.1006/jmre.2002.2541

Dyce, E. J. (1935). 1,987,893. US patent.

Fabri, D., Williams, M. A. K., \& Halstead, T. K. (2005). Water T2 relaxation in sugar solutions. Carbohydrate Research, 340(5), 889-905. https://doi.org/10.1016/j.carres.2005.01.034

Gleiter, R. A., Horn, H., \& Isengard, H. D. (2006). Influence of type and state of crystallisation on the water activity of honey. Food Chemistry, 96(3), 441-445. https://doi.org/10.1016/j.foodchem.2005.03.051

Gonnet, M. (1994). La cristallisation dirigée des miels: actualization des méthodes de travail et avantages liés a 
cette pratique tecnologique. Abeilles \& Fleurs, 430, 12.

385

Hartel, R. W. (1993). Controlling Sugar Crystallization in Food Products. Food Technology, 47(11), 99-107. https://doi.org/10.1080/10408399109527541

Hills, B. (1998). Magnetic Resonance Imaging in Food Science. (Whiley, Ed.). London: Wiley.

Hills, B. P., \& Pardoe, K. (1995). Proton and deuterium NMR studies of the glass transition in a 10\% watermaltose solution. Journal of Molecular Liquids, 63(3), 229-237. https://doi.org/10.1016/01677322(95)00796-D

Hills, B. P., Wright, K. M., \& Belton, P. S. (1989). Proton N.M.R. Studies of chemical and diffusive exchange in carbohydrate systems. Molecular Physics, 67(6), 1309-1326. https://doi.org/10.1080/00268978900101831

Hubert, M., Rousseeuw, P. J., \& Vanden Branden, K. (2005). ROBPCA: A new approach to robust principal component analysis. Technometrics, 47(1), 64-79. https://doi.org/Doi 10.1098/004017004000000563

Iaccheri, E., Laghi, L., Cevoli, C., Berardinelli, A., Ragni, L., Romani, S., \& Rocculi, P. (2015). Different analytical approaches for the study of water features in green and roasted coffee beans. Journal of Food Engineering, 146. https://doi.org/10.1016/j.jfoodeng.2014.08.016

Karasu, S., Toker, O. S., Yilmaz, M. T., Karaman, S., \& Dertli, E. (2015). Thermal loop test to determine structural changes and thermal stability of creamed honey: Rheological characterization. Journal of Food Engineering, 150, 90-98. https://doi.org/10.1016/j.jfoodeng.2014.10.004

Laghi, L., Cremonini, M. A., Placucci, G., Sykora, S., Wright, K., \& Hills, B. (2005). A proton NMR relaxation study of hen egg quality. Magnetic Resonance Imaging, 23(3). https://doi.org/10.1016/j.mri.2004.12.003

Mauro, M. A., Dellarosa, N., Tylewicz, U., Tappi, S., Laghi, L., Rocculi, P., \& Rosa, M. D. (2016). Calcium and ascorbic acid affect cellular structure and water mobility in apple tissue during osmotic dehydration in sucrose solutions. Food Chemistry, 195, 19-28. https://doi.org/10.1016/j.foodchem.2015.04.096

Meiboom, S., \& Gill, D. (1958). Modified spin-echo method for measuring nuclear relaxation times. Review of Scientific Instruments. https://doi.org/10.1063/1.1716296

Petracci, M., Laghi, L., Rimini, S., Rocculi, P., Capozzi, F., \& Cavani, C. (2014). Chicken breast meat marinated with increasing levels of sodium bicarbonate. Journal of Poultry Science, 51(2), 0130079. https://doi.org/10.2141/jpsa.0130079

Petracci, M., Laghi, L., Rocculi, P., Rimini, S., Panarese, V., Cremonini, M. A., \& Cavani, C. (2012). The use of 
sodium bicarbonate for marination of broiler breast meat. Poultry Science, 91(2), 526-534. https://doi.org/10.3382/ps.2011-01753

R Development Core Team, R. (2011). R: A Language and Environment for Statistical Computing. $R$ Foundation for Statistical Computing (Vol. 1). https://doi.org/10.1007/978-3-540-74686-7

Ribeiro, R. de O. R., Mársico, E. T., Carneiro, C. D. S., Monteiro, M. L. G., Júnior, C. C., \& Jesus, E. F. O. De. (2014). Detection of honey adulteration of high fructose corn syrup by Low Field Nuclear Magnetic Resonance (LF 1H NMR). Journal of Food Engineering, 135, 39-43. https://doi.org/10.1016/j.jfoodeng.2014.03.009

Ribeiro, R. de O. R., Mársico, E. T., Carneiro, C. da S., Monteiro, M. L. G., Conte Júnior, C. A., Mano, S., \& de Jesus, E. F. O. (2014). Classification of Brazilian honeys by physical and chemical analytical methods and low field nuclear magnetic resonance (LF 1H NMR). LWT - Food Science and Technology, 55(1), 90-95. https://doi.org/10.1016/j.lwt.2013.08.004

Serra-Bonvehì, J. (1974). La cristallisation du miel. Facteurs qui l'affecten. Bullettin Technique Apicole, 54(13), $37-48$.

Venir, E., Spaziani, M., \& Maltini, E. (2010). Crystallization in "Tarassaco" Italian honey studied by DSC. Food Chemistry, 122(2), 410-415. https://doi.org/10.1016/j.foodchem.2009.04.012

Venturi, L., Rocculi, P., Cavani, C., Placucci, G., Dalla Rosa, M., \& Cremonini, M. A. (2007). Water absorption of freeze-dried meat at different water activities: A multianalytical approach using sorption isotherm, differential scanning calorimetry, and nuclear magnetic resonance. Journal of Agricultural and Food Chemistry, 55(26), 10572-10578. https://doi.org/10.1021/jf072874b

Zamora, M. C., \& Chirife, J. (2006). Determination of water activity change due to crystallization in honeys from Argentina. Food Control, 17(1), 59-64. https://doi.org/10.1016/j.foodcont.2004.09.003 


\section{Figures Captions}

437 Figure 1: Relationship between enthalpy and storage time for static (continuous lines) and dynamic 438 (dashed lines) crystallization, for FC (points), MC (squares) and SC (triangles) samples.

439 Fig. 2. Relative concentration and $\mathrm{T}_{2}$ of the two protons populations non-exchangeable and 440 exchangeable with water, as calculated from $\mathrm{T}_{2}$ weighted curves obtained by TD-NMR on samples 441 stored statically (empty symbols) or dynamically (filled symbols) for fast (black circles), medium (dark 442 gray squares) and slow (light gray triangles) crystalizing samples. To ease visual inspection of the data, 443 trend dashed lines have been added for samples stored statically, while only samples at $\mathrm{T}_{0}$ and $\mathrm{T}_{\mathrm{f}}$ have 444 been represented for samples stored dynamically.

445 Figure 3: rPCA model calculated on the centered and scaled points of the $\mathrm{T}_{2}$ weighted TD-NMR 446 signals. A) Scoreplot of samples stored statically (empty symbols) or dynamically (filled symbols) for 447 fast (black circles), medium (dark gray squares) and slow (light gray triangles) crystalizing samples. To 448 ease visual inspection of the data, for samples stored statically trend dashed lines have been added, 449 while for samples stored dynamically only data from $\mathrm{T}_{0}$ and $\mathrm{T}_{\mathrm{f}}$ have been represented. B) Example of $450 \mathrm{~T}_{2}$ weighted TD-NMR signals, registered on FC samples at the beginning (black) and at the end (gray) 451 of the storage period. C) Correlation between the points of the signals and their importance over PC1 


\section{Declaration of Interest Statement}

\section{Declaration of interests}

$\bigotimes$ The authors declare that they have no known competing financial interests or personal relationships that could have appeared to influence the work reported in this paper.

$\square$ The authors declare the following financial interests/personal relationships which may be considered as potential competing interests: 
Table 1. Composition $(\mathrm{g} / 100 \mathrm{~g})$ of samples.

\begin{tabular}{|c|c|c|c|c|c|c|c|}
\hline & Fructose & Glucose & Sucrose & Turanose & Maltose & Water & $\mathrm{F} / \mathrm{G}$ \\
\hline $\mathrm{FCs}$ & 39.2 & 36.2 & $<0.5$ & 0.8 & 1.4 & 17.7 & 1.08 \\
\hline MCs & 38.6 & 32.5 & 0.5 & 0.8 & 1.1 & 16.5 & 1.19 \\
\hline $\mathrm{SCs}$ & 42.8 & 31.0 & $<0.5$ & 1.4 & 0.7 & 16.8 & 1.38 \\
\hline $\mathrm{FCd}$ & 39.0 & 36.4 & $<0.5$ & 0.9 & 1.2 & 16.0 & 1.07 \\
\hline $\mathrm{MCd}$ & 39.7 & 32.9 & $<0.5$ & 1.3 & 1.1 & 17.5 & 1.21 \\
\hline $\mathrm{SCd}$ & 38.0 & 27.1 & $<0.5$ & 1.1 & 0.6 & 17.3 & 1.4 \\
\hline
\end{tabular}

Table 2. Main features of the crystallization kinetics observed through melting enthalpy.

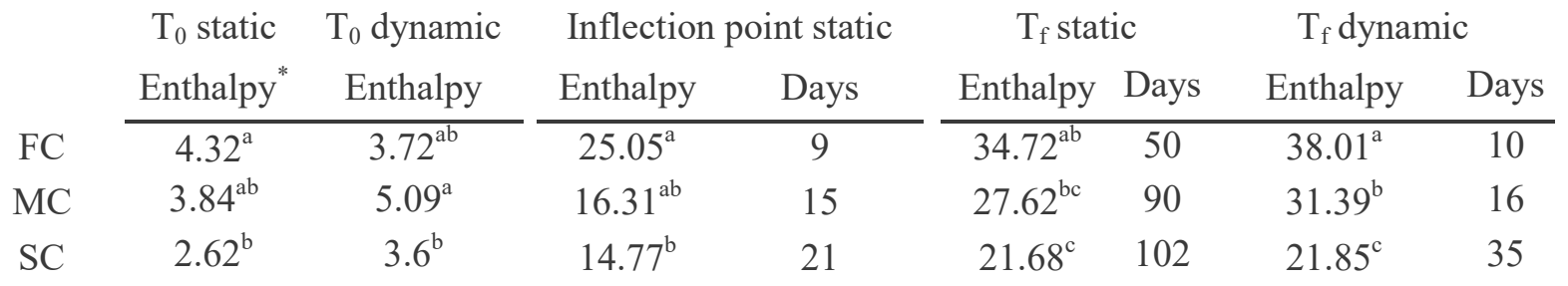

"Enthalpy is expressed in J/g. At each time-point, different letters indicate significant differences between samples. For readability, data dispersion is not indicated.

Table 3. For TD-NMR data, main features of the trends evidenced by the non-parametric fitting (dashed lines of figure 2).

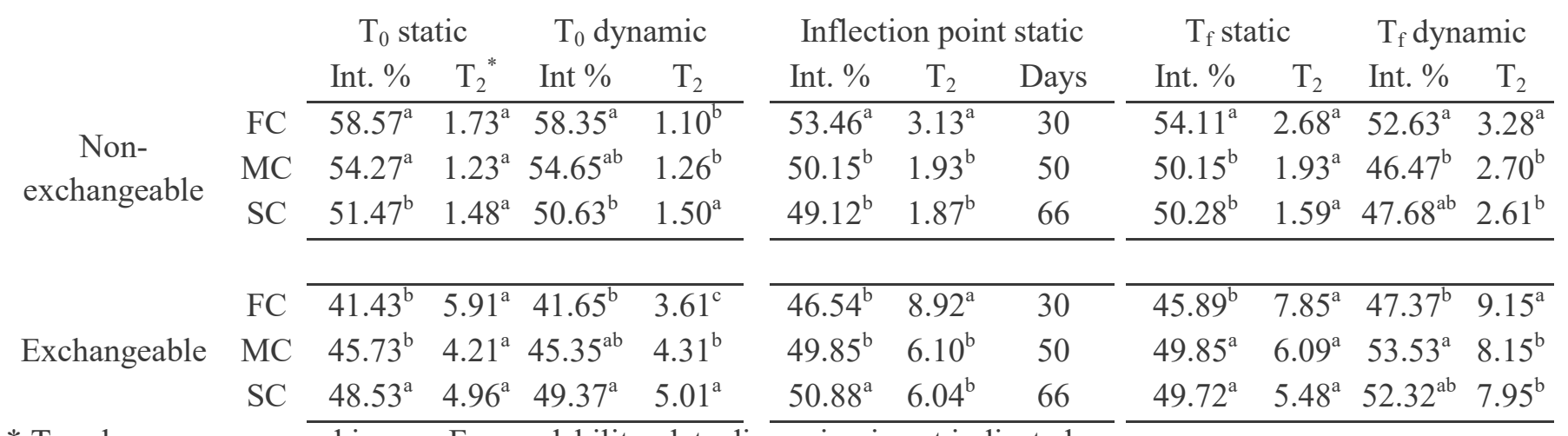

* $\mathrm{T}_{2}$ values are expressed in ms. For readability, data dispersion is not indicated. 


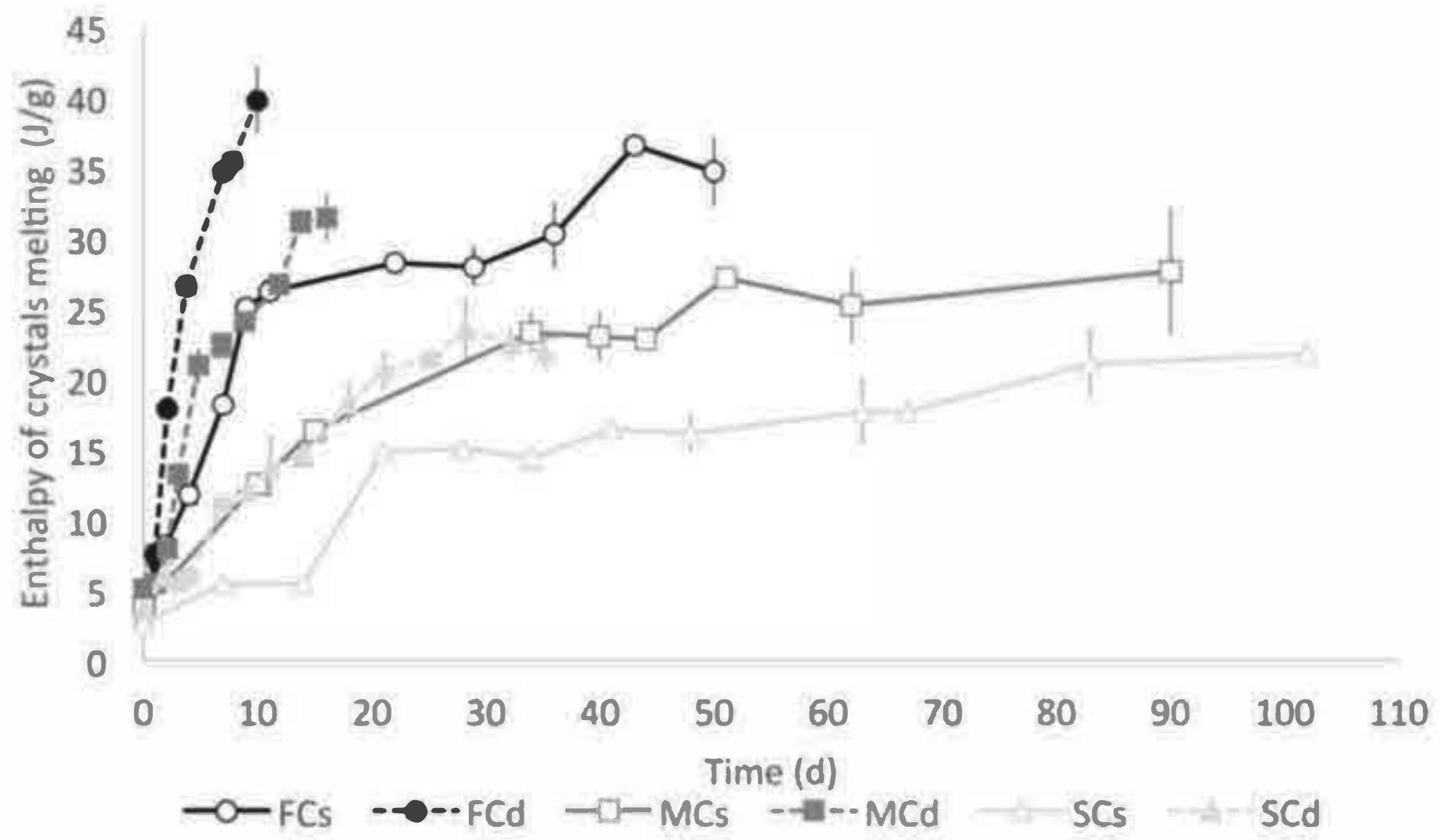


Figure(s)
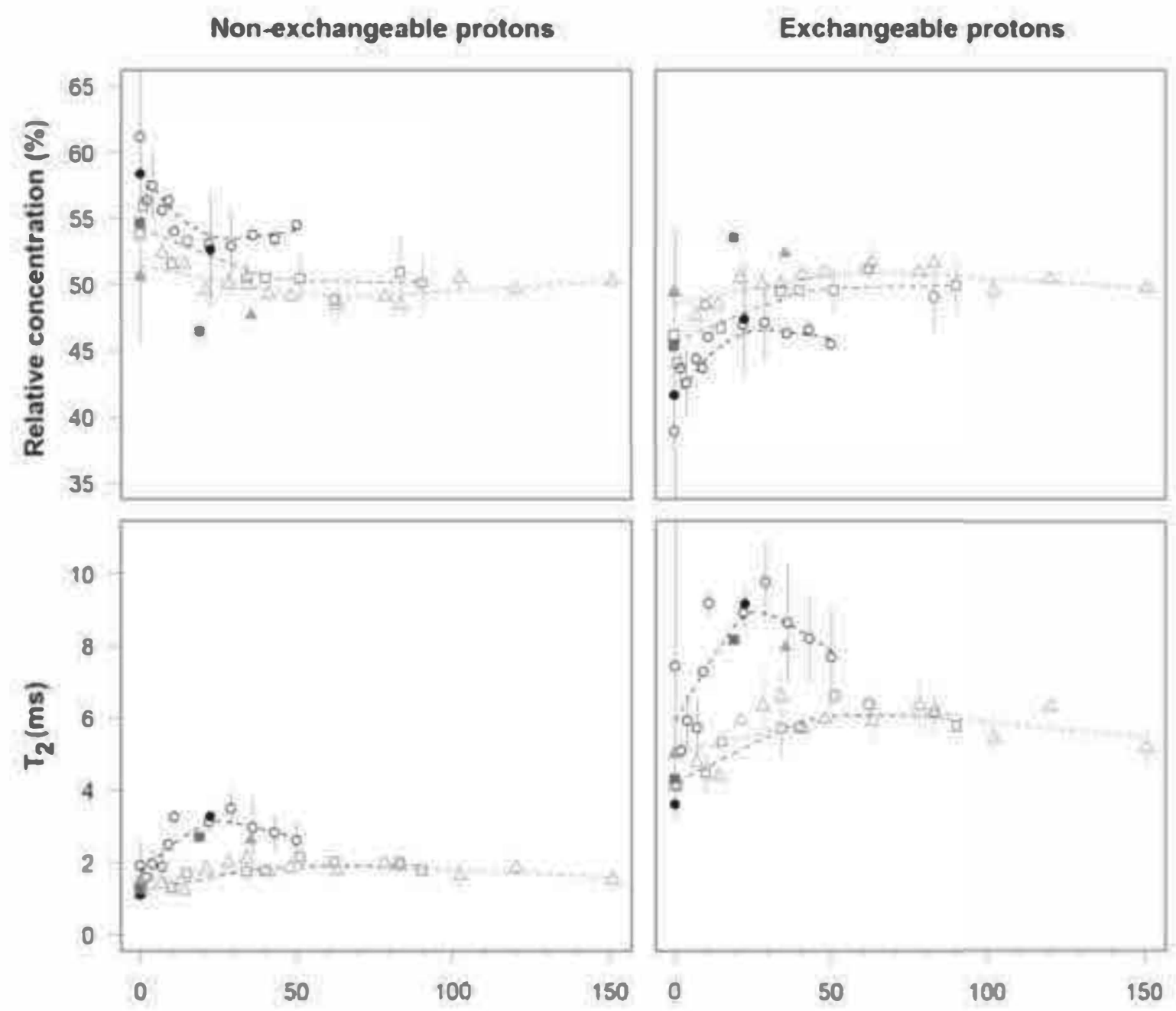

Time (d) 

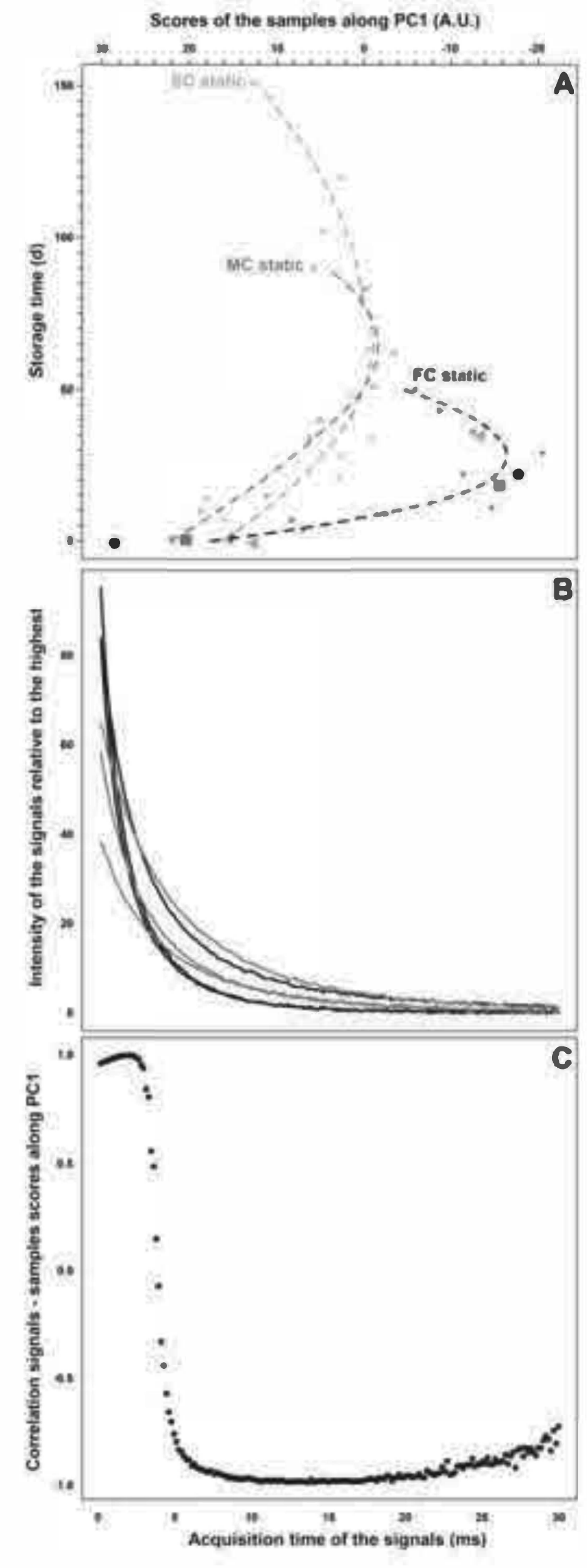

Figure(s) 